\title{
Unraveling the Mesoscopic Character of Quantum Dots in Nanophotonics
}

\author{
P. Tighineanu, ${ }^{*}$ A. S. Sørensen, S. Stobbe, and P. Lodahl ${ }^{\dagger}$ \\ Niels Bohr Institute, University of Copenhagen, Blegdamsvej 17, DK-2100 Copenhagen, Denmark \\ (Received 29 August 2014; published 19 June 2015)
}

\begin{abstract}
We provide a microscopic theory for semiconductor quantum dots that explains the pronounced deviations from the prevalent point-dipole description that were recently observed in spectroscopic experiments on quantum dots in photonic nanostructures. The deviations originate from structural inhomogeneities generating a large circular quantum current density that flows inside the quantum dot over mesoscopic length scales. The model is supported by the experimental data, where a strong variation of the multipolar moments across the emission spectrum of quantum dots is observed. Our work enriches the physical understanding of quantum dots and is of significance for the fields of nanophotonics, quantum photonics, and quantum-information science, where quantum dots are actively employed.
\end{abstract}

DOI: 10.1103/PhysRevLett.114.247401

PACS numbers: 78.67.Hc, 42.50.Ct, 75.75.-c, 78.47.-p

Semiconductor quantum dots (QDs) are compatible with semiconductor technology and exhibit bright optical transitions, which renders them promising single-photon sources for a range of solid-state quantum-optical devices [1]. For instance, strong coupling between a QD and a cavity photon [2] or near-unity coupling to a photoniccrystal waveguide have been demonstrated [3]. Furthermore, QDs have been proposed as the enabling part of highly efficient solar cells [4] and as central nodes in future quantum-information systems with entangled stationary and flying quantum bits [5]. It was observed recently that, surprisingly, QDs can break the widely used dipole approximation and the deviations were explained by a phenomenologically defined mesoscopic moment [6]. Current band-structure models of QDs cannot explain these mesoscopic quantum effects, whose physical origin has been unclear so far. Here we develop an extension to the effective-mass theory for nanostructures. We find that QDs possess large strain-induced structural inhomogeneities at the crystal-lattice level, which generate a quantum current density flowing along a curved path inside the QD, cf. Fig. 1(b). This results in large electric and magnetic moments leading to light-matter interaction of both electric and magnetic character $[7,8]$. The mesoscopic moment can be tuned over orders of magnitude by controlling the size and shape of QDs. The developed theory is used to describe and explain the experimental data on time-resolved spectroscopy of QDs positioned near a dielectric interface in a Drexhage geometry [9] (cf. Fig. 1), where a strong variation of the mesoscopic moment with emission energy is found for the case of $\operatorname{In}(\mathrm{Ga})$ As self-assembled QDs. Our findings can be immediately used for engineering nanophotonic environments that maximize the coupling to the currentdensity pattern of the QD.

A central quantity describing the optical transition from the excited state $\Psi_{e}$ to the ground state $\Psi_{g}$ of a QD is the dipole moment $\boldsymbol{\mu}=\left(e / m_{0}\right)\left\langle\Psi_{g}|\hat{\mathbf{p}}| \Psi_{e}\right\rangle$, where $e$ and $m_{0}$ are the elementary charge and electron mass, respectively. We consider the $x$-polarized dipole moment of the exciton $\boldsymbol{\mu}=\mu \hat{\mathbf{x}}$, as sketched in Fig. 1, where $\hat{\mathbf{x}}$ is the Cartesian unit vector. Until recently, $\mu$ was the only QD property used to describe the interaction with light. Recent experimental studies of spontaneous emission of QDs at nanoscale proximity to a mirror [6] revealed surprisingly strong deviations from the dipole theory, which were explained by a large multipolar moment $\Lambda=\left(e / m_{0}\right)\left\langle\Psi_{g}\left|x \hat{p}_{z}\right| \Psi_{e}\right\rangle$. It was subsequently found that $\mu$ and $\Lambda$ enable probing electric and magnetic vacuum fluctuations simultaneously [7]. While the microscopic origin of $\mu$ is well known to be
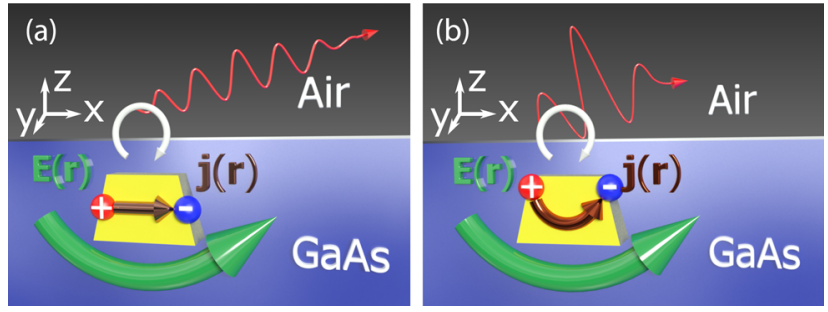

FIG. 1 (color online). Unraveling the mesoscopic character of QDs in the vicinity of a GaAs-air interface. The presence of the interface breaks the parity symmetry of the environment in the $z$ direction. Since reflections occur at the interface (the circular white arrow), the imaginary part of the electric field $\mathbf{E}(\mathbf{r})$ generated by the electric-dipole component, which triggers spontaneous emission, inherits this lack of symmetry and is curved (indicated by the green arrow). (a) In the dipole approximation, the QD current $\mathbf{j}(\mathbf{r})$ (brown arrow) perceives only the parallel component but not the out-of-plane component (the "curvature") of the electromagnetic field at its position. (b) In In(Ga)As self-assembled QDs, the current density flows along a curved path that resembles the shape of the field environment, thereby exchanging energy more efficiently with it. As a consequence, the spontaneous-emission decay rate is enhanced and the photons (red arrows) are emitted at a faster rate compared to the case in (a). 
related to $\Psi_{g}$ and $\Psi_{e}$ having opposite parity symmetry, the mesoscopic moment $\Lambda$ has so far been employed as a phenomenological parameter with an unclear microscopic description. In the present work we develop a theory explaining that $\Lambda$ primarily originates from structural inhomogeneities occurring in the nanoscopic crystal lattice of the QD. This is markedly different from existing theories [10-17], which fail to reproduce the large values of $\Lambda$ observed experimentally because they neglect the atomistic nature of QDs. The structural inhomogeneities are related to a change in the periodicity of the underlying crystal lattice in the QD. Since Bloch functions with different periodicities cannot remain in phase throughout the QD, this necessarily leads to a phase gradient and a resulting current in the growth direction of the QD, which gives rise to the mesoscopic moment, cf. Fig. 1(b). To obtain an optimized light-matter coupling, the electromagnetic environment must be shaped similarly to the flow of the QD current, as sketched in Fig. 1(b), where we exemplify the case of a GaAs-air interface.

Previous experiments reported the measurements of spontaneous-emission decay rates for ensembles of QDs that are placed at different distances to a GaAs-air interface $[18,19]$. Figure 2(a) displays the data that were used to reliably extract the dipole moment by exploiting the data points recorded at distances above $75 \mathrm{~nm}$ [18]. A systematic deviation from the dipole theory was found at distances below $\sim 75 \mathrm{~nm}$, which was speculated to be a result of enhanced loss processes at the etched interfaces. The deviations can be explained by the contributions from the mesoscopic moment $\Lambda$ to the light-matter interaction strength. The theory of Ref. [7] quantitatively reproduces the functional dependence observed in the experiment for all emission energies, as exemplified in Fig. 2(a), see the Supplemental Material [20] for details. The extracted
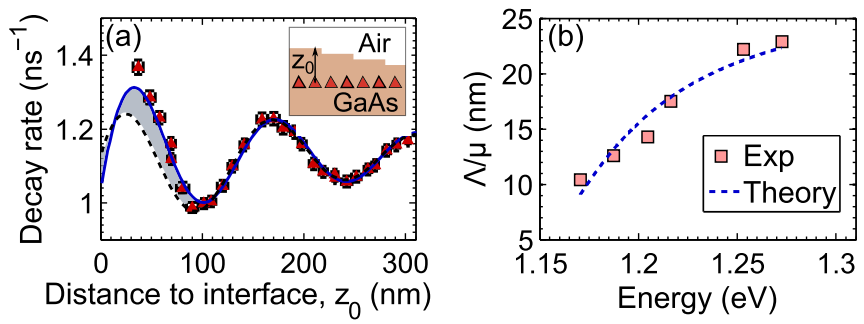

FIG. 2 (color online). Observation of deviations from dipole theory for QDs near an interface. (a) Measured decay rates versus distance $z_{0}$ to the GaAs-air interface (data points) at an energy of $1.27 \mathrm{eV}$ (wavelength $\lambda_{0} \sim 975 \mathrm{~nm}$ ). The dipole (multipolar) theory is indicated by the black dashed (blue solid) line. A refractive index $n=3.5$ of GaAs was used. The inset is a schematic illustrating the sample geometry. (b) Extracted mesoscopic strength $\Lambda / \mu$ over the emission spectrum of QDs (red squares) along with the prediction of the theoretical model (blue dashed line) assuming that the QDs have a fixed in-plane size and only the height varies, cf. the Supplemental Material [20]. mesoscopic strength $\Lambda / \mu$ increases with emission energy and varies from 10 to $23 \mathrm{~nm}$ over the inhomogeneously broadened emission spectrum, cf. Fig. 2(b), and is successfully explained by our microscopic QD theory, which is presented in the following.

Numerous band structure models have been proposed for QDs ranging from continuum approaches [21], which discard the QD atomistic nature and consider only the macroscopic potential, to atomistic models [22], which simulate the contribution of every single atom comprising the QD. In the present work, it suffices to consider the simplest band structure model, i.e., the two-band effectivemass theory. The QD single-particle wave function can be written as a product of a periodic Bloch function $u(\mathbf{r})$, which captures the properties on the length scale of the crystal unit cell, and a slowly varying envelope $\psi(\mathbf{r})$ that inherits the size and symmetry of the QD potential, i.e., $\Psi_{i}(\mathbf{r})=u_{i}(\mathbf{r}) \psi_{i}(\mathbf{r})$ with $i=\{g, e\}$ corresponding to the ground or excited state, respectively. The standard textbook approach for evaluating the transition dipole moment $\mu$ is to assume that the envelope function varies slowly over a unit cell so that $\mu$ can be written as a product of the Bloch matrix element $p_{c v}$ and a three-dimensional overlap integral between the envelope functions, i.e., $\mu=\left(e / m_{0}\right)\left\langle u_{g} \psi_{g}\left|\hat{p}_{x}\right| u_{e} \psi_{e}\right\rangle \approx\left(e / m_{0}\right) p_{c v}\left\langle\psi_{g} \mid \psi_{e}\right\rangle$, where $p_{c v}=V_{\mathrm{UC}}^{-1} \int_{\mathrm{UC}} d^{3} \mathbf{r} u_{g}^{*} \hat{p}_{x} u_{e}$ is given by an integral over the unit cell with $V_{\mathrm{UC}}$ being the unit-cell volume. Importantly, the large mesoscopic strength $\Lambda / \mu$ observed experimentally [see Fig. 2(b)] cannot be reproduced by a similar calculation, which leads to

$$
\begin{aligned}
\Lambda= & \frac{e}{m_{0}}\left[\left\langle\psi_{g}|x| \psi_{e}\right\rangle\left\langle u_{g}\left|\hat{p}_{z}\right| u_{e}\right\rangle_{\mathrm{UC}}+\left\langle\psi_{g} \mid \psi_{e}\right\rangle\left\langle u_{g}\left|x \hat{p}_{z}\right| u_{e}\right\rangle_{\mathrm{UC}}\right. \\
& \left.+\left\langle\psi_{g}\left|x \hat{p}_{z}\right| \psi_{e}\right\rangle\left\langle u_{g} \mid u_{e}\right\rangle_{\mathrm{UC}}+\left\langle\psi_{g}\left|\hat{p}_{z}\right| \psi_{e}\right\rangle\left\langle u_{g}|x| u_{e}\right\rangle_{\mathrm{UC}}\right],
\end{aligned}
$$

where \langle\rangle$_{\mathrm{UC}} \equiv V_{\mathrm{UC}}^{-1} \int_{\mathrm{UC}} \mathrm{d}^{3} \mathbf{r}$ denotes integration over a unit cell. The first three contributions vanish for symmetry reasons. The fourth contribution is vanishingly small and does not scale with the QD size: for Gaussian envelopes allowing for realistic mutual displacements of 1-2 nm between the electron and the hole in the growth direction (note that the integral vanishes in the absence of such a displacement) we estimate $\Lambda / \mu \sim 10^{-4} \mathrm{~nm}$. This suggests that the large mesoscopic strength $\Lambda / \mu \sim 10-20 \mathrm{~nm}$ observed experimentally cannot be explained solely by the envelope wave functions. In the following, we show that structural gradients at the nanoscopic crystal-lattice length scale can explain the effect.

It is often assumed that solid-state emitters have a homogeneous chemical composition, which justifies the use of bulk-material Bloch functions, while only the slowly varying envelopes describe the properties of the nanostructure. This assumption works excellently for quantum 
wells and lattice-matched QDs, where the wave functions are confined to a chemically homogeneous region of space. InAs QDs are grown by self-assembly induced by strain relaxation, which unavoidably leads to the generation of structural gradients at the crystal-lattice level. In particular, large lattice-constant shifts were observed in the growth direction of QDs [23,24], which limits the applicability of the standard effective-mass formalism. An atomistic theory would generally be required to describe the mesoscopic light-matter interaction. Remarkably, the essential physics can be captured by only a minor extension of the effectivemass theory. We assume that the lattice periodicity changes at a certain position $z=z_{T}$ along the QD height by an amount $\Delta a_{l}=110 \mathrm{pm}$ at a central value $a_{l}=605 \mathrm{pm}$ as found experimentally in Ref. [23], see Fig. 3(a). This corresponds to a relative lattice-constant shift of $18 \%$, which is strain induced and substantially larger than the lattice-constant mismatch between InAs and GaAs of $7 \%$. We note that, in general, the lattice periodicity changes twice: first it is expanded at the QD base [GaAs-In $(\mathrm{Ga}) \mathrm{As}$ transition] before being shrunk back at the QD tip [ $\operatorname{In}(\mathrm{Ga})$ As-GaAs transition]. Since the exciton is spatially confined near the tip where the indium concentration is highest [24], we only consider the second transition region. The Bloch functions change periodicity as well, cf. Fig. 3(b), and we model this by expanding them in a Fourier series with a position-dependent lattice wave vector $k_{l}(z)$ :

$$
\begin{aligned}
& u_{g}(\mathbf{r})=\sum_{m} a_{m}(y, z) \sin \left[m k_{l}(z) x\right], \\
& u_{e}(\mathbf{r})=\sum_{n} b_{n}(y, z) \cos \left[n k_{l}(z) x\right] .
\end{aligned}
$$

This ansatz ensures opposite parity of the conduction- and valence-band Bloch functions along $x$. Furthermore, we implicitly assume the shape of the Bloch functions to remain the same, and only their periodicity to vary spatially. Now we return to the evaluation of the mesoscopic moment and separate the slowly and rapidly varying contributions as

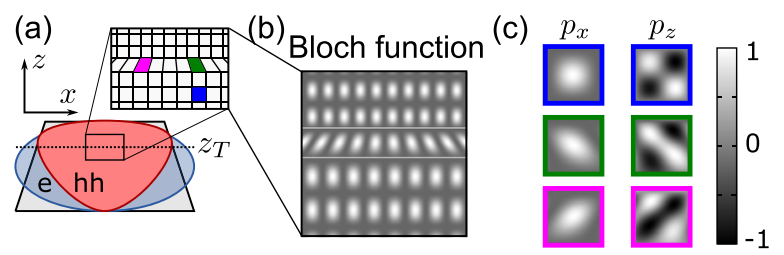

FIG. 3 (color online). Sketch illustrating the microscopic model for mesoscopic QDs. (a) The atomic lattice inside the QD is assumed to change periodicity at the position $z=z_{T}$. (b) Sketch of how the Bloch function $u_{g}^{2}$ of the atomic lattice varies spatially inside the QD. (c) Illustration of the matrix elements $\left\langle p_{x}\right\rangle \equiv\left\langle u_{g}\left|\hat{p}_{x}\right| u_{e}\right\rangle$ and $\left\langle p_{z}\right\rangle \equiv\left\langle u_{g}\left|\hat{p}_{z}\right| u_{e}\right\rangle$ for the three colored unit cells in (a). The symmetry of the integrand is broken in the transition region around $z=z_{T}$, giving rise to pronounced mesoscopic effects.

$$
\Lambda=\frac{e}{m_{0}} \sum_{q=1}^{N} \psi_{g}^{*}\left(\mathbf{R}_{q}\right) X_{q} \psi_{e}\left(\mathbf{R}_{q}\right) \int_{\mathrm{UC}} d^{3} \mathbf{r} u_{g}^{*}(\mathbf{r}) \hat{p}_{z} u_{e}(\mathbf{r})
$$

where $\mathbf{R}_{q}$ denotes the position of the $q$ th unit cell and $N$ is the total number of unit cells in the QD. In a homogeneous region of the QD [the blue unit cell in Fig. 3(a)], the unit-cell integrand of Eq. (3) is odd in the $x$ and $z$ directions, cf. Fig. 3(c), which leads to a vanishing integral. However, in the transition region around $z=z_{T}$ strong gradients are present, which destroy the parity of the integrand [see the pink and green unit cells in Figs. 3(a) and 3(c)] and generate a substantial contribution to $\Lambda$.

With the ansatz in Eq. (2) we first compute the dipole Bloch matrix element $\left\langle\hat{p}_{x}\right\rangle$ and then evaluate $\Lambda$, which can be expressed in terms of $\left\langle\hat{p}_{x}\right\rangle$ because the $x$ and $z$ derivatives of the Bloch functions yield similar results, cf. Eqs. (2)-(3). The resulting expression for $\Lambda / \mu$ reads

$$
\frac{\Lambda}{\mu}=\frac{1}{k_{l}} \frac{\left\langle\psi_{g}\left|x^{2}\left[\partial_{z} k_{l}(z)\right]\right| \psi_{e}\right\rangle}{\left\langle\psi_{g} \mid \psi_{e}\right\rangle} .
$$

The mesoscopic strength scales quadratically with the in-plane size of the QD, $\Lambda / \mu \sim L_{r}^{2}$, because the term $\left\langle\psi_{g}\left|x^{2}\left[\partial_{z} k_{l}(z)\right]\right| \psi_{e}\right\rangle$ contains the variance of the exciton wave function in the $x$ direction. Moreover, it increases with decreasing QD height, $\Lambda / \mu \sim L_{z}^{-1}$, since in shallow QDs the relative importance of the lattice-constant transition region is increased. We use Eq. (4) to model the spectral dependence of $\Lambda / \mu$, see Fig. 2(b), where only the height of QDs is assumed to vary across the spectrum while the in-plane size remains constant, as justified in the Supplemental Material [20]. By mapping the quantization energy to the QD size, we are able to extract a QD height that varies from 11 to $3 \mathrm{~nm}$ across the inhomogeneously broadened spectrum, cf. Fig. 4(a), which agrees well with the values obtained from atomic-force microscopy measurements [19]. The details of the calculation are given in the Supplemental Material [20]. From Fig. 2(b) we conclude that QDs with larger emission energy have larger mesoscopic strengths because they are shallow so that a large part of the excitonic wave function is affected by the lattice inhomogeneity.

Equation (4) is the most general expression for $\Lambda / \mu$ that can be simplified in order to obtain an intuitive analytical expression. We consider the particular case of in-plane rotationally symmetric Gaussian slowly varying envelopes and obtain

$$
\frac{\Lambda}{\mu}=-\frac{\Delta a_{l}}{a_{l}} \sqrt{\frac{1+\xi_{z}}{4 \pi}} \frac{\sigma_{r}^{2}}{\sigma_{z}},
$$

where $\sigma_{z}$ is the height (HWHM) of the electron envelope, $\sigma_{r}$ the QD radius, $\Delta a_{l} / a_{l}$ the relative lattice-constant shift and $\xi_{z} \approx 5$ is the ratio between the electron and hole 
effective masses [25]. We plot the mesoscopic strength as a function of the in-plane radius for three fixed heights in Fig. 4(b). The largest mesoscopic strengths are achieved for shallow and wide (disk-shaped) QDs. For instance, taking a relatively extreme yet experimentally realistic case of a height $2 \sigma_{z}=2 \mathrm{~nm}$ and radius $\sigma_{r}=30 \mathrm{~nm}$ yields a mesoscopic strength as large as $\Lambda / \mu \simeq 120 \mathrm{~nm}$, which is an order of magnitude larger than the values observed in experiments so far. In such QDs mesoscopic effects would dominate the light-matter interaction strength. For instance, if placed in front of a silver mirror it would exhibit a Purcell factor nearly 100 times larger than a point-dipole source. Such QDs may also be extremely efficient at interfacing electric and magnetic degrees of freedom in structures that conserve parity symmetry, such as photonic-crystal cavities and waveguides, owing to the substantial increase of second-order light-matter-interaction processes that are weak for current $\operatorname{In}(\mathrm{Ga})$ As QDs.
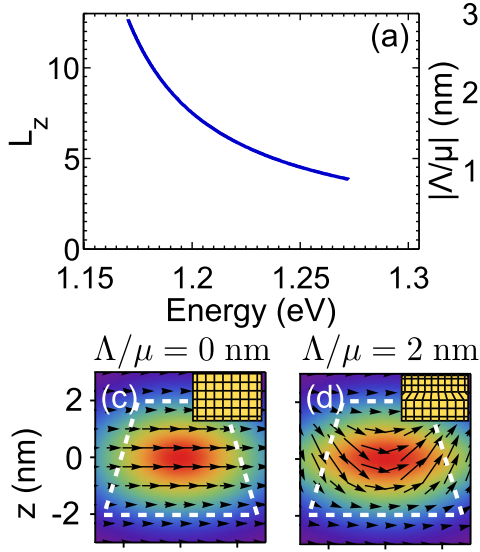

$\begin{array}{lll}-5 & 0 & 5\end{array}$

$\begin{array}{lllllll}-5 & 0 & 5 & -15 & -10 & -5\end{array}$ $\Lambda / \mu=25 \mathrm{~nm}$

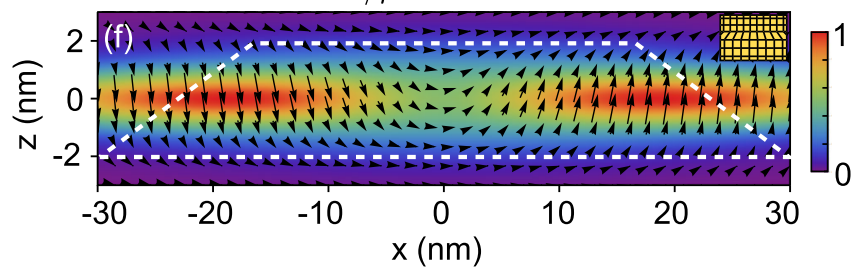

FIG. 4 (color online). The mesoscopic strength and the associated current density running through the QD. (a) Spectral dependence of the QD height predicted by the theoretical model. (b) Mesoscopic strength as a function of the in-plane size of the QD for three fixed QD heights. (c)-(f) Plots of quantum current densities for various QD geometries. (c) Homogeneous crystal lattice where the current flow is uniform and points in the direction of the dipole moment. (d) Inhomogeneous lattice for a QD radius of $5 \mathrm{~nm}$ giving rise to a nonuniform current flow following a curved path. The QD height is $4 \mathrm{~nm}$. (e),(f) Same as (d) but for QD radii of 10 and $20 \mathrm{~nm}$, respectively. In (c)-(f), both the length of the arrows and the color scale indicate the magnitude of the flow and the direction of the arrows indicates the pointwise direction of the flow. The dashed white line sketches the position and orientation of the QD.
Knowledge about the quantum-mechanical wave functions allows computing the current density $\mathbf{j}_{\mathrm{QD}}(\mathbf{r})$ flowing through the QD. We define the latter by comparing the interaction Hamiltonian $\hat{H}_{\text {int }}=\left(e / m_{0}\right) \mathbf{A} \cdot \hat{\mathbf{p}}$, where $\mathbf{A}$ is the vector potential, to the classical particle-field interaction Hamiltonian $H_{\text {int }}=\mathbf{A}(\mathbf{r}) \cdot \mathbf{j}(\mathbf{r})$ [9]. The quantummechanical current density can therefore be written as $\mathbf{j}_{\mathrm{QD}}(\mathbf{r})=\left(e / m_{0}\right) \Psi_{g}^{*}(\mathbf{r}) \hat{\mathbf{p}} \Psi_{e}(\mathbf{r})$ or

$$
\mathbf{j}_{\mathrm{QD}}(\mathbf{r})=\frac{e}{m_{0}}\left[\Psi_{g}^{*} \hat{p}_{x} \Psi_{e} \hat{\mathbf{x}}+\Psi_{g}^{*} \hat{p}_{z} \Psi_{e} \hat{\mathbf{z}}\right] .
$$

In the following, Gaussian slowly varying envelopes are used to model the current density. In QDs with a homogeneous crystal lattice and thus negligible mesoscopic moment, the current density flows only along the direction of the dipole moment because there are no gradients in the $z$ direction [see Fig. 4(c)]. Note that for simplicity we ignore the modulation of $\mathbf{j}_{\mathrm{QD}}$ by the periodic Bloch functions in Fig. 4. The presence of lattice inhomogeneities changes the flow dramatically because strong gradients in the $z$ direction arise. The current density flows along a curved path as illustrated in Figs. 4(d)-4(f), conferring pronounced mesoscopic properties to QDs. The wider the QD is, the sharper the transverse oscillations of the current are and the larger $\Lambda / \mu$ is. This effect offers the possibility to enhance (diminish) the light-matter interaction by placing QDs in environments where the electric vacuum field exhibits gradients with the same (opposite) sign; see also Fig. 1. Aside from the local light-matter coupling strength, other degrees of freedom could be potentially tailored by exploiting the mesoscopic interaction, such as the photonemission directionality or polarization.

We emphasize that the mesoscopic character of $\operatorname{In}(\mathrm{Ga}) \mathrm{As}$ QDs could play an important role in any photonic nanostructure that breaks parity symmetry along the QD height. A particular example is the field of quantum plasmonics, where QDs are placed in strong field gradients [26] to achieve large Purcell factors [27] and strong quantum nonlinearities [28]. Another example concerns the control of spontaneous emission in photonic metamaterials [29], in which the presented theory could be employed for tailoring the electric and magnetic coupling to the QD. In contrast, the dipole theory is highly robust in parity-symmetric environments such as photonic-crystal cavities [2] and waveguides [3] with QDs that are placed in the center of the membrane structure because $\mu$ and $\Lambda$ couple to modes with different parity and do not interfere [7].

The present work concerns mesoscopic effects in selfassembled $\operatorname{In}(\mathrm{Ga})$ As QDs, but the theory is generally valid for all QDs grown by strain relaxation and thus exhibiting distortions of the underlying crystal lattice, such as $\mathrm{Ge} / \mathrm{Si}$ self-assembled QDs [30]. An interesting question pertains to the existence of such effects in other types of widely studied solid-state quantum emitters, such as colloidal [31] or monolayer-fluctuation [32] QDs. The presented 
theoretical framework in conjunction with experiments in photonic environments lacking parity symmetry may provide a valuable route to study such effects. The reported deviations from dipole theory found for $\operatorname{In}(\mathrm{Ga})$ As QDs are caused by the combination of two effects: the QD size $(\sim 20 \mathrm{~nm})$ is comparable to the wavelength of light $\lambda_{0} / 2 \pi n \sim 45 \mathrm{~nm}$, and the QD wave functions break parity symmetry due to the lattice distortion.

In conclusion, we have developed an extension to the effective-mass theory for nanostructures that successfully explains the large mesoscopic strengths of $\operatorname{In}(\mathrm{Ga})$ As QDs observed experimentally. We find the effect to be governed by structural inhomogeneities at the level of the nanoscopic crystal lattice, which generate a mesoscopic current density that oscillates along a curved path inside the QD. Our work is expected to be of significance for solid-state quantum electrodynamics and quantum-information processing, where efficient quantum interfaces between QDs and light are exploited.

We thank P.T. Kristensen and M. L. Andersen for valuable discussions. We gratefully acknowledge financial support from the Danish Council for Independent Research (natural sciences and technology and production sciences), the European Research Council (ERC consolidator grants "ALLQUANTUM" and "QIOS"), and the Carlsberg Foundation.

*petru.tighineanu@nbi.ku.dk

†lodahl@nbi.ku.dk; http://www.quantum-photonics.dk/

[1] P. Lodahl, S. Mahmoodian, and S. Stobbe, Rev. Mod. Phys. 87, 347 (2015).

[2] T. Yoshie, A. Scherer, J. Hendrickson, G. Khitrova, H. Gibbs, G. Rupper, C. Ell, O. Shchekin, and D. Deppe, Nature (London) 432, 200 (2004).

[3] M. Arcari, I. Söllner, A. Javadi, Hansen S. Lindskov, S. Mahmoodian, J. Liu, H. Thyrrestrup, E. H. Lee, J. D. Song, S. Stobbe, and P. Lodahl, Phys. Rev. Lett. 113, 093603 (2014).

[4] H. A. Atwater and A. Polman, Nat. Mater. 9, 205 (2010).

[5] W. Gao, P. Fallahi, E. Togan, J. Miguel-Sanchez, and A. Imamoglu, Nature (London) 491, 426 (2012).

[6] M. L. Andersen, S. Stobbe, A. S. Sørensen, and P. Lodahl, Nat. Phys. 7, 215 (2011).

[7] P. Tighineanu, M. L. Andersen, A. S. Sørensen, S. Stobbe, and P. Lodahl, Phys. Rev. Lett. 113, 043601 (2014).

[8] I. Sersic, C. Tuambilangana, T. Kampfrath, and A. F. Koenderink, Phys. Rev. B 83, 245102 (2011).
[9] L. Novotny and B. Hecht, Principles of Nano-Optics (Cambridge University Press, Cambridge, England, 2012).

[10] S. V. Goupalov, Phys. Rev. B 68, 125311 (2003).

[11] M. Sugawara, Phys. Rev. B 51, 10743 (1995).

[12] A. Thränhardt, C. Ell, G. Khitrova, and H. M. Gibbs, Phys. Rev. B 65, 035327 (2002).

[13] K. Jun Ahn and A. Knorr, Phys. Rev. B 68, 161307 (2003).

[14] J. R. Zurita-Sánchez and L. Novotny, J. Opt. Soc. Am. B 19, 1355 (2002).

[15] J. R. Zurita-Sánchez and L. Novotny, J. Opt. Soc. Am. B 19, 2722 (2002).

[16] S. Stobbe, P. T. Kristensen, J. E. Mortensen, J. M. Hvam, J. Mørk, and P. Lodahl, Phys. Rev. B 86, 085304 (2012).

[17] P. T. Kristensen, J. E. Mortensen, P. Lodahl, and S. Stobbe, Phys. Rev. B 88, 205308 (2013).

[18] J. Johansen, S. Stobbe, I. S. Nikolaev, T. Lund-Hansen, P. T. Kristensen, J. M. Hvam, W. L. Vos, and P. Lodahl, Phys. Rev. B 77, 073303 (2008).

[19] S. Stobbe, J. Johansen, P. T. Kristensen, J. M. Hvam, and P. Lodahl, Phys. Rev. B 80, 155307 (2009).

[20] See Supplemental Material at http://link.aps.org/ supplemental/10.1103/PhysRevLett.114.247401 for details regarding the analysis of the experimental data from Fig. 2.

[21] J. Luttinger and W. Kohn, Phys. Rev. 97, 869 (1955).

[22] G. Bester, J. Phys. Condens. Matter 21, 023202 (2009).

[23] H. Eisele, A. Lenz, R. Heitz, R. Timm, M. Dähne, Y. Temko, T. Suzuki, and K. Jacobi, J. Appl. Phys. 104, 124301 (2008).

[24] D. Bruls, J. Vugs, P. Koenraad, H. Salemink, J. Wolter, M. Hopkinson, M. Skolnick, F. Long, and S. Gill, Appl. Phys. Lett. 81, 1708 (2002).

[25] Y. Y. Peter and M. Cardona, Fundamentals of Semiconductors: Physics and Materials Properties (Springer, New York, 2010).

[26] M. Pfeiffer, K. Lindfors, C. Wolpert, P. Atkinson, M. Benyoucef, A. Rastelli, O. G. Schmidt, H. Giessen, and M. Lippitz, Nano Lett. 10, 4555 (2010).

[27] X.-W. Chen, M. Agio, and V. Sandoghdar, Phys. Rev. Lett. 108, 233001 (2012).

[28] D. E. Chang, A. S. Sørensen, E. A. Demler, and M. D. Lukin, Nat. Phys. 3, 807 (2007).

[29] K. Tanaka, E. Plum, J. Y. Ou, T. Uchino, and N. I. Zheludev, Phys. Rev. Lett. 105, 227403 (2010).

[30] G. Costantini, A. Rastelli, C. Manzano, R. Songmuang, O. Schmidt, K. Kern, and H. von Känel, Appl. Phys. Lett. 85, 5673 (2004).

[31] B. Dabbousi, J. Rodriguez-Viejo, F. V. Mikulec, J. Heine, H. Mattoussi, R. Ober, K. Jensen, and M. Bawendi, J. Phys. Chem. B 101, 9463 (1997).

[32] D. Gammon, E. Snow, B. Shanabrook, D. Katzer, and D. Park, Science 273, 87 (1996). 